\title{
ALGORITHMS FOR THE CHINESE POSTMAN PROBLEM ON MIXED NETWORKS
}

\author{
Wen Lea Pearn ${ }^{1} \dagger$ and C. M. Liu ${ }^{2} \ddagger$ \\ ${ }^{1}$ Department of Industrial Engineering \& Management, National Chiao Tung University, Hsinchu, \\ Taiwan, R.O.C. and ${ }^{2}$ Kaohsiung Military School, Kaohsiung, Taiwan, R.O.C.
}

(Received August 1993; in revised form May 1994)

\begin{abstract}
Scope and Purpose-Given an undirected street network, the celebrated Chinese postman problem (CPP) is that of finding a shortest postman tour covering all the edges (streets) in the network. The Chinese postman problem on mixed networks (MCPP), is an extension of the CPP, in which some streets from the network are allowed to be traversed in both directions, and others may be traversed in one specified direction only. Applications of the MCPP include: routing of newspaper or mail delivery vehicles, parking meter coin collection or household refuse collection vehicles, street sweepers, snow plows, and school buses; spraying roads with salt, inspection of electric power lines, or oil or gas pipelines, and reading electric meters. The MCPP has been shown to be NP-complete, and heuristic solution procedures have been proposed to solve the problem approximately. The purpose of this paper is to review the existing solution procedures, and present two modifications of the existing methods to obtain better problem solutions.

Abstract-The Chinese postman problem on mixed networks (MCPP), is a practical generalization of the classical Chinese postman problem (CPP), which has many real-world applications. The MCPP has been shown to be NP-complete. Therefore, it is difficult to solve the problem exactly. For this reason, heuristic solution procedures have been proposed to solve the problem approximately. In this paper, we first review two existing solution procedures proposed by Edmonds and Johnson [Math. Progr. 5, 88-124 (1973)], and Frederickson [J. Assoc. Comput. Mach. 26, 538-554 (1979)], then introduce two new algorithms to solve the MCPP near optimally. The proposed new algorithms are tested and compared with the existing solution procedures. Computational results showed that the two new algorithms significantly outperformed the existing solution procedures.
\end{abstract}

\section{INTRODUCTION}

The Chinese postman problem on mixed networks (MCPP), is an interesting generalization of the classical Chinese postman problem (CPP), in which the underlying (street) network is mixed. On mixed networks, some streets (edges) are allowed to be traversed in both directions (two-way) with equal distance, and others (arcs) may be traversed in one specified direction only (one-way). Such a generalization, of course, reflects real-life situations more directly. In particular, for cities with narrow streets, or where there is a need for traffic flow control.

The MCPP may be briefly defined as follows. We are given a street network $G=(V, E, A)$ with $V$ representing a set of nodes, $E$ representing a set of undirected edges (two-way streets), and $A$ representing a set of directed arcs (one-way streets). For each edge $(i, j) \in E$, we have two non-negative distances: $d(i, j)$ for the direction from node $i$ to node $j$, and $d(j, i)$ for the direction from node $j$ to node $i$ with $d(j, i)=d(i, j)$. For each arc $(i, j) \in A$, we have a non-negative distance $d(i, j)$ for the direction from node $i$ to node $j$, and $d(j, i)$ is set to infinity in this case. Then, the MCPP is to find a postman tour, starting from the depot, traversing each edge and arc in $E \cup A$ at least once, and

†Dr W. L. Pearn received his Ph.D. degree from the University of Maryland at College Park, Maryland. His area of interest include network optimization, and quality management. He has been with AT\&T Bell Laboratories, and currently he is a professor of Operations Research in the Department of Industrial Engineering and Management at National Chiao Tung University, Taiwan, R.O.C.

${ }_{\ddagger} \mathrm{Mr}$ C. M. Liu received his M.S. degree from the Department of Industrial Engineering and Management, National Chiao Tung University. Currently, he is an instructor at Kaohsiung Military School, R.O.C. 
returning to the same depot with total distance traversed minimized. Clearly, if $A=\varnothing$, then the MCPP reduces to the classical CPP.

The solution to the CPP (defined on undirected networks) may be obtained efficiently using a polynomial-time bounded matching algorithm owing to Edmonds and Johnson [1]. But, the MCPP (defined on mixed networks) has been shown to be NP-complete [2]. Christofides et al. [3] presented an integer programming formulation of the problem, and developed an exact algorithm to solve the MCPP optimally. The algorithm is essentially based on a branch-and-bound algorithm using Lagrangean relaxations. Minieka [4] presented a transformation converting the MCPP into a flow with gains problem, which allows the MCPP to be solved optimally via linear programming and cutting plane techniques. Unfortunately, both approaches are computationally inefficient; only problems of small or moderate size may be solved optimally. Because of the problem's complexity, several heuristic solution procedures have been proposed to solve the MCPP approximately $[1,3,5,6]$. In this paper, we first review the existing solution procedures, then present two new algorithms to solve the problem approximately.

\section{REVIEW OF EXISTING SOLUTION PROCEDURES}

Real-world applications directly related to the MCPP include: routing of newspaper [7] or mail delivery vehicles [8], parking meter or household refuse collection vehicles [9], street sweepers, snow plows and school buses [10]; spraying roads with salt $[11,12]$, inspection of electric power lines, or oil or gas pipelines, and reading electric meters [13].

An undirected network is called even if every node in the network has even degree. A directed network is called symmetric if for every node, $i$, in the network, $\operatorname{deg}^{+}(i)=\operatorname{deg}^{-}(i)$, where $\operatorname{deg}^{+}(i)=$ the number of arcs directed out of node $i$, and $\operatorname{deg}^{-}(i)=$ the number of arcs directed into node $i$. If the network is even (or symmetric), then a postman route can be constructed from the network without repeating any edges (or arcs). Details of this tour construction procedure may be found in Ref. [14].

Edmonds and Johnson [1] presented an algorithm to solve the MCPP approximately. The algorithm essentially consists of two phases. Phase I converts the original network into an even one by treating each (directed) arc as an (undirected) edge. Phase II transforms the network (obtained from the first phase) into a symmetric one. Since the transformed symmetric network is not necessarily maintained even, the postman tour may not be constructed. Frederickson [5] modified the algorithm by adding a new phase (Phase III). The new phase recovers the resulting network back to even so that a postman tour can be constructed. This algorithm was referred to as MIXED1. Frederickson [5] further considered an alternative approach, which is essentially the reverse of the Edmonds and Johnson [1] approach. The reverse approach first makes the network symmetric, then even. The reverse approach has been referred to as MIXED2. Other solution procedures such as those developed by Brucker [6], and Christofides et al. [3] are essentially the same as MIXED1 and MIXED2 and, therefore, will not be discussed here.

\subsection{MIXEDI algorithm}

Phase I. convert $G$ into an even network.

Step 1. Let $G^{*}$ be the new network obtained from $G$ with all arc directions ignored.

Step 2. Solve the CPP over the new network $G^{*}$ using the matching algorithm owing to Edmonds and Johnson [1]. Let $Z(E)$ be the set of edges, and $Z(A)$ the set of arcs obtained from the matching. We also let $E_{1}=E \cup Z(E), A_{1}=A \cup Z(A)$. Then, $G_{1}=\left(V, E_{1}, A_{1}\right)$ is even.

Phase II. transform $G_{1}$ into a symmetric network.

Step 1. Construct a new network $G_{2}=\left(V, A_{2}\right)$ with arc costs, arc capacities, and node demands defined as:

for each edge $(i, j) \in E_{1}$, create four new arcs in $A_{2}$ including

(a) one copy of $(i, j)$ with cost $d(i, j)$ and infinite flow capacity,

(b) one copy of $(j, i)$ with cost $d(i, j)$ and infinite flow capacity, 
(c) one copy of $(i, j)$, denote it as $(i, j)^{\prime}$, with cost zero and flow capacity 1 ,

(d) one copy of $(j, i)$, denote it as $(j, i)^{\prime}$, with cost zero and flow capacity 1 ;

for each arc $(k, l) \in A_{1}$, create one copy of $(k, l)$ in $A_{2}$, with cost $d(k, l)$ and infinite flow capacity.

for each node $i \in V$, define

(a) demand $=\left\{\operatorname{deg}^{+}(i)\right.$ in $\left.G_{2}\right\}-\left\{\operatorname{deg}^{-}(i)\right.$ in $\left.G_{2}\right\}$ if deg ${ }^{+}(i)$ in $G_{2}>\operatorname{deg}^{-}(i)$ in $G_{2}$,

(b) supply $=\left\{\operatorname{deg}^{-}(i)\right.$ in $\left.G_{2}\right\}-\left\{\operatorname{deg}^{+}(i)\right.$ in $\left.G_{2}\right\}$ if $G_{2}>\operatorname{deg}^{+}(i)$ in $G_{2}$, where $\operatorname{deg}^{+}(i)=$ the number of arcs incident out of node $i$, and $\operatorname{deg}^{-}(i)=$ the number of arcs incident into node $i$.

Step 2. Find the minimum-cost flow over the network $G_{2}$. Let $Y_{i j}, Y_{j i}, Y_{i j^{\prime}}, Y_{j i^{\prime}}$, and $Y_{k l}$ be the number of flow units among the $\operatorname{arcs}(i, j),(j, i),(i, j)^{\prime},(j, i)^{\prime}$, and $(k, l)$, respectively.

Step 3. Construct a symmetric network $G_{3}=\left(V, E_{3}, A_{3}\right)$. Initially, we set $E_{3}=\varnothing$, and $A_{3}=A_{1}$.

(a) if $Y_{i j^{\prime}}+Y_{j i^{\prime}}=1$, put $Y_{i j^{\prime}}$ copies of $\operatorname{arc}(i, j)$ and $Y_{j i^{\prime}} \operatorname{copies}$ of $\operatorname{arc}(j, i)$ in $A_{3}$,

(b) if $Y_{i j^{\prime}}+Y_{j i^{\prime}} \neq 1$, put one copy of edge $(i, j)$ in $E_{3}$,

(c) put $Y_{i j}$ copies of $\operatorname{arc}(i, j)$ and $Y_{j i}$ copies of $\operatorname{arc}(j, i)$ in $A_{3}$,

(d) put $Y_{k l}$ copies of arc $(k, l)$ in $A_{3}$.

Phase III. Recover $G_{3}$ back to even [5].

Let $A^{\prime}$ be the set of artificial arcs generated from Phase II, and $E_{4}=E_{3}$. Identify cycles from $A^{\prime} \cup E_{4}$ consisting of alternating paths in $A^{\prime}$ and $E_{4}$, with each path anchored at each end by an odd node from $G_{3}$. In finding such cycles, directions of the arcs on paths from $A^{\prime}$ should be ignored. As the cycles cover all odd nodes from $G_{3}$, directions will be arbitrarily assigned to the cycles, and arcs on the cycles will be either duplicated or deleted depending upon if the direction of the cycle is the same as the original direction of the arcs in $A^{\prime}$, and edges will be oriented. Consequently, all the nodes from $G_{3}$ will be even and symmetric (for further descriptions, see Frederickson [5]). Denote the resulting network as $G_{4}$.

Phase I of the algorithm applies the minimum-cost matching algorithm to convert the original network into an even one. Phase II of the algorithm applies the minimum-cost flow algorithm to transform the resulting network into a symmetric one. And Phase III of the algorithm recovers the resulting symmetric network back to even. Frederickson [5] showed that the computational complexity of the algorithm is $O\left(\max \left\{|V|^{3},|A|(\max \{|A|,|E|\})^{2}\right)\right\}$, where once again $|V|=$ the number of nodes, $|A|=$ the number of arcs, and $|E|=$ the number of edges from the network. Frederickson [5] further showed that the performance of this algorithm, in the worst case, has a bound of 2, that is, (MIXED1 Solution)/(Optimal Solution) $\leqslant 2$, and the bound is approachable.

Example 1. Consider the network depicted in Fig. 1 with six nodes, six edges, and two arcs. The

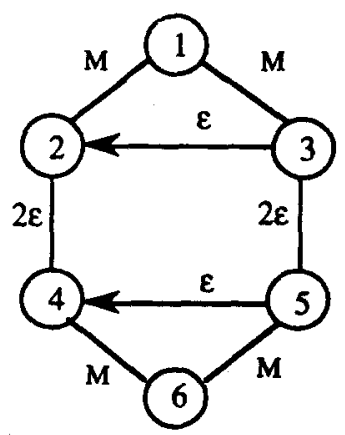

Fig. 1. The MCPP network described in Example 1. 
associated edge and arc lengths are defined as follows: for edges, $d(1,2)=d(1,3)=d(4,6)=d(5,6)=M$, $d(2,4)=d(3,5)=2 \varepsilon$; for arcs, $d(3,2)=d(5,4)=\varepsilon$, where $M>\varepsilon>0$. It is easy to verify that the solution obtained by MIXED1 contains artificial edges and arcs $\{(1,3),(1,3),(2,1),(2,1),(3,2),(5,4)\}$, and has a total length of $8 M+8 \varepsilon$, while the optimal solution has a total length of $4 M+10 \varepsilon$. Thus, we have $($ MIXED1 Solution) $/($ Optimal Solution $)=(8 M+8 \varepsilon) /(4 M+10 \varepsilon) \rightarrow 2$, as $M \gg \varepsilon$.

\subsection{MIXED2 algorithm}

Phase I. Transform $G$ into a symmetric network.

Step 1. Construct a new network $G_{1}=\left(V, A_{1}\right)$ with arc costs, arc capacities, and node demand defined as:

for each edge $(i, j) \in E$, create four new arcs in $A_{1}$ including

(a) one copy of $(i, j)$ with cost $d(i, j)$ and infinite flow capacity,

(b) one copy of $(j, i)$ with cost $d(i, j)$ and infinite flow capacity,

(c) one copy of $(i, j)$, denote it as $(i, j)^{\prime}$, with cost zero and flow capacity 1 ,

(d) one copy of $(j, i)$, denote it as $(j, i)^{\prime}$, with cost zero and flow capacity 1 ;

for each arc $(k, l) \in A$, create one copy of $(k, l)$ in $A_{1}$, with cost $d(k, l)$ and infinite flow capacity.

for each node $i \in V$, define

(a) demand $=\left\{\operatorname{deg}^{+}(i)\right.$ in $\left.G_{1}\right\}-\left\{\operatorname{deg}^{-}(i)\right.$ in $\left.G_{1}\right\}$ if $\operatorname{deg}^{+}(i)$ in $G_{1}>\operatorname{deg}^{-}(i)$ in $G_{1}$,

(b) supply $=\left\{\operatorname{deg}^{-}(i)\right.$ in $\left.G_{1}\right\}-\left\{\operatorname{deg}^{+}(i)\right.$ in $\left.G_{1}\right\}$ if $\operatorname{deg}^{-}(i)$ in $G_{1}>\operatorname{deg}^{+}(i)$ in $G_{1}$, where $\operatorname{deg}^{+}(i)=$ the number of arcs incident out of node $i$, and $\operatorname{deg}^{-}(i)=$ the number of arcs incident into node $i$.

Step 2. Find the minimum-cost flow over the network $G_{1}$. Let $Y_{i j}, Y_{j i}, Y_{i j}, Y_{j i}$, and $Y_{k l}$ be the number of flow units among the arcs $(i, j),(j, i),(i, j)^{\prime},(j, i)^{\prime}$, and $(k, l)$, respectively.

Step 3. Construct a symmetric network $G_{2}=\left(V, E_{2}, A_{2}\right)$. Initially, we set $E_{2}=\varnothing$, and $A_{2}=A$.

(a) if $Y_{i j^{\prime}}+Y_{j i^{\prime}}=1$, put $Y_{i j^{\prime}}$ copies of $\operatorname{arc}(i, j)$ and $Y_{j i^{\prime}}$ copies of $\operatorname{arc}(j, i)$ in $A_{2}$,

(b) if $Y_{i j^{\prime}}+Y_{j i^{\prime}} \neq 1$, put one copy of edge $(i, j)$ in $E_{2}$,

(c) put $Y_{i j}$ copies of $\operatorname{arc}(i, j)$ and $Y_{j i}$ copies of $\operatorname{arc}(j, i)$ in $A_{2}$,

(d) put $Y_{k l}$ copies of $\operatorname{arc}(k, l)$ in $A_{2}$.

Phase II. Convert $G_{2}$ into an Eulerian network.

Step 1. Solve the CPP over the subnetwork of $G$ consisting of $E_{2}$ and the associated nodes using the matching algorithm of Edmonds and Johnson [1].

Step 2. Let $Z\left(E_{2}\right)$ be the set of edges obtained from the matching, and $E_{3}=E_{2} \cup Z\left(E_{2}\right)$, $A_{3}=A_{2}$. The Eulerian network $G_{3}=\left(V, E_{3}, A_{3}\right)$ is the desired solution.

Clearly, the computational complexity of MIXED2 is the same as that of MIXED1, i.e. $O\left(\max \left\{|V|^{3}, \mid A\{\mid(\max |A|,|E|\})^{2}\right\}\right)$. The bound on the worst-case performance of MIXED2 is also the same as that for MIXED1, and the bound is approachable.

Example 2. Consider the network depicted in Fig. 2 with eight nodes, six edges, and six arcs. The associated edge and arc lengths are defined as follows: for edges, $d(1,9)=d(2,9)=d(3,8)=d(5,8)=$ $d(4,9)=d(6,9)=M$; for $\operatorname{arcs}, d(1,2)=d(3,4)=d(5,6)=\varepsilon, d(2,3)=d(4,5)=d(6,1)=2 \varepsilon$, where $M>\varepsilon>0$. Clearly, the network is symmetric. Therefore, we may proceed with Phase II of the algorithm directly. It is easy to verify that the following artificial edges $\{(1,7),(7,2),(3,9),(9,4),(5,8),(8,6)\}$ may be generated and the MIXED2 solution is $12 M+9 \varepsilon$, while the optimal solution is $6 M+12 \varepsilon$. Hence, we have (MIXED2 Solution) $/($ Optimal Solution) $=(12 M+9 \varepsilon) /(6 M+12 \varepsilon) \rightarrow 2$, as $M \gg \varepsilon$.

To improve the solution, Frederickson [5] considered a mixed-strategy approach. The mixed-strategy approach first calls MIXED1 and MIXED2 algorithms to generate two complete MCPP solutions, then selects the best of the two. We refer to this approach as MIXED1-2. 


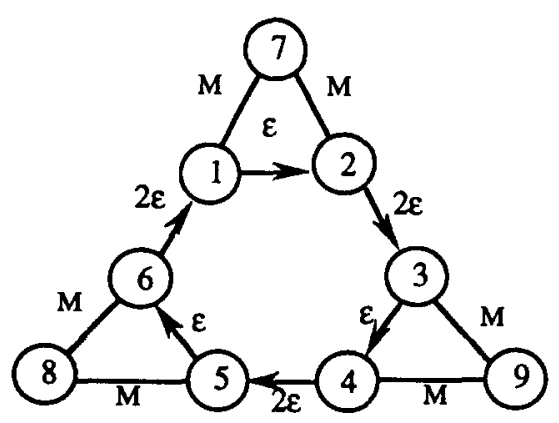

Fig. 2. The MCPP network described in Example 2.

Frederickson [5] showed that the worst-case bound for this approach, is $5 / 3$. In fact, if we apply MIXED1-2 algorithm to example 1 , then a solution of minimum $\{8 \mathrm{M}+8 \varepsilon, 4 M+10 \varepsilon\}=4 M+10 \varepsilon$ may be obtained, which is optimal. Similarly, if we apply MIXED1-2 algorithm to example 2, then a solution of minimum $\{6 M+12 \varepsilon, 12 M+9 \varepsilon\}=6 M+12 \varepsilon$ may be obtained, which is also optimal.

\subsection{A comparison}

To compare MIXED1 and MIXED2 algorithms. We generated 60 problems. Some of the test problems are dense networks, and others are sparse. These problems were generated by arbitrarily linking pairs of nodes so that a connected network is formed. The lengths of edges and arcs were also arbitrarily generated. These problems are described in the following with $|V|$ representing the number of nodes, $|E|$ representing the number of edges, $|A|$ representing the number of arcs, and $P$ representing the ratio $|A| /(|A|+|E|)$ in the original MCPP network.

Set A: 5 problems; $|V|=10 ; 2 \leqslant|E| \leqslant 5,13 \leqslant|A| \leqslant 25,70 \%<P \leqslant 100 \%$.

Set B: 5 problems; $|V|=20 ; 2 \leqslant|E| \leqslant 19,45 \leqslant|A| \leqslant 113,70 \%<P \leqslant 100 \%$.

Set C: 5 problems; $|V|=30 ; 10 \leqslant|E| \leqslant 55,115 \leqslant|A| \leqslant 235,70 \%<P \leqslant 100 \%$.

Set D: 5 problems; $|V|=35 ; 7 \leqslant|E| \leqslant 53,238 \leqslant|A| \leqslant 302,70 \%<P \leqslant 100 \%$.

Set $A^{\prime}: 5$ problems; $|V|=10 ; 7 \leqslant|E| \leqslant 16,10 \leqslant|A| \leqslant 12,40 \%<P \leqslant 70 \%$.

Set $B^{\prime}$ : 5 problems; $|V|=20 ; 28 \leqslant|E| \leqslant 61,40 \leqslant|A| \leqslant 54,40 \%<P \leqslant 70 \%$.

Set $C^{\prime}: 5$ problems; $|V|=30 ; 74 \leqslant|E| \leqslant 165,86 \leqslant|A| \leqslant 191,40 \%<P \leqslant 70 \%$.

Set $\mathrm{D}^{\prime}$ : 5 problems; $|V|=35 ; 88 \leqslant|E| \leqslant 186,120 \leqslant|A| \leqslant 274,40 \%<P \leqslant 70 \%$.

Set $\mathrm{A}^{\prime \prime}: 5$ problems; $|V|=10 ; 10 \leqslant|E| \leqslant 18,3 \leqslant|A| \leqslant 5,0 \%<P \leqslant 40 \%$.

Set $B^{\prime \prime}: 5$ problems; $|V|=20 ; 55 \leqslant|E| \leqslant 101,5 \leqslant|A| \leqslant 34,0 \%<P \leqslant 40 \%$.

Set $C^{\prime \prime}: 5$ problems; $|V|=30 ; 108 \leqslant|E| \leqslant 216,33 \leqslant|A| \leqslant 72,0 \%<P \leqslant 40 \%$.

Set $\mathrm{D}^{\prime \prime}: 5$ problems; $|V|=35 ; 182 \leqslant|E| \leqslant 266,44 \leqslant|A| \leqslant 131,0 \%<P \leqslant 40 \%$.

Table 1 presents the solutions generated by MIXED1 and MIXED2 on the 60 test problems. The lower bounds were obtained by solving the CPP over the (undirected) network derived from the original one by ignoring all the arc directions, which is to be used as a convenient reference point for assessing the accuracy of the heuristic solutions. In Table 2, the performance of MIXED1 and MIXED2 are compared. The results indicated that: (1) MIXED1 significantly outperformed MIXED2 for problems with $0 \%<P \leqslant 70 \%$, as MIXED1 received 33 best solutions out of 40 test problems (which is $82.5 \%$ ), (2) MIXED2 significantly outperformed MIXED1 for problems with $70 \%<P \leqslant 100 \%$ (most streets are one-way), as MIXED2 received 18 best solutions out of 20 test problems (which is 90\%), and (3) MIXED1 performed extremely well on problems with $0 \%<P \leqslant 40 \%$ (most streets are two-way), as the algorithm received 19 problem optimal solutions out of 20 test problems (which is $95 \%$ ).

\section{NEW ALGORITHMS}

Recall that MIXED1 first applies minimal-cost matching then minimum-cost flow algorithms. Both procedures generate artificial arcs. In some cases, these artificial arcs have the same direction 
Wen Lea Pearn and C. M. Liu

Table 1. Solution of the 60 test problems (underlines indicate the best solutions)

\begin{tabular}{|c|c|c|c|c|c|c|}
\hline $\begin{array}{l}\text { Problem } \\
\text { number }\end{array}$ & $\begin{array}{l}\text { Number } \\
\text { of nodes }\end{array}$ & $\begin{array}{l}\text { Number } \\
\text { of edges }\end{array}$ & $\begin{array}{l}\text { Number } \\
\text { of arcs }\end{array}$ & $\begin{array}{l}\text { Lower } \\
\text { bound }\end{array}$ & MIXED1 & MIXED2 \\
\hline A1 & 10 & 5 & 13 & 120 & 192 & 177 \\
\hline A2 & 10 & 2 & 24 & 170 & 284 & 274 \\
\hline A3 & 10 & 4 & 13 & 100 & 186 & 154 \\
\hline A4 & 10 & 6 & 24 & 200 & 282 & 264 \\
\hline A5 & 10 & 3 & 15 & 110 & 162 & 137 \\
\hline B1 & 20 & 7 & 59 & 409 & 613 & $\frac{157}{572}$ \\
\hline B2 & 20 & 15 & 57 & 426 & 658 & $\overline{619}$ \\
\hline B3 & 20 & 16 & 71 & 507 & 633 & 615 \\
\hline B4 & 20 & 6 & 113 & 636 & 824 & 814 \\
\hline B5 & 20 & 2 & 112 & 604 & 806 & 787 \\
\hline $\mathrm{Cl}$ & 30 & 30 & 183 & 1186 & 1354 & $1 \overline{349}$ \\
\hline $\mathrm{C} 2$ & 30 & 23 & 198 & 1153 & 1337 & $\overline{1324}$ \\
\hline $\mathrm{C} 3$ & 30 & 52 & 235 & 1587 & 1787 & $\frac{769}{1769}$ \\
\hline $\mathrm{C} 4$ & 30 & 56 & 148 & 1182 & 1230 & $\overline{1261}$ \\
\hline C5 & 30 & 24 & 115 & 801 & $\overline{1075}$ & 1028 \\
\hline D1 & 35 & 57 & 323 & 2133 & 2195 & $\overline{2225}$ \\
\hline D2 & 35 & 36 & 291 & 1837 & 2045 & 2030 \\
\hline D3 & 35 & 12 & 273 & 1505 & 1861 & $\overline{1798}$ \\
\hline D4 & 35 & 10 & 174 & 994 & 1436 & $\overline{1331}$ \\
\hline D5 & 35 & 7 & 302 & 1537 & 1727 & 1686 \\
\hline $\mathbf{A}^{\prime} 1$ & 10 & 15 & 10 & 185 & 185 & 207 \\
\hline$A^{\prime} 2$ & 10 & 7 & 11 & 110 & $\frac{180}{180}$ & 157 \\
\hline$A^{\prime} 3$ & 10 & 12 & 12 & 167 & 213 & 205 \\
\hline$A^{\prime} 4$ & 10 & 16 & 11 & 209 & 229 & $\overline{254}$ \\
\hline$A^{\prime} 5$ & 10 & 10 & 17 & 181 & 217 & 220 \\
\hline $\mathbf{B}^{\prime} 1$ & 20 & 28 & 40 & 485 & $\overline{545}$ & 558 \\
\hline$B^{\prime} 2$ & 20 & 61 & 54 & 757 & $\overline{763}$ & 782 \\
\hline $\mathbf{B}^{\prime} 3$ & 20 & 42 & 51 & 631 & $\overline{685}$ & 671 \\
\hline$B^{\prime} 4$ & 20 & 36 & 41 & 547 & 585 & 579 \\
\hline $\mathbf{B}^{\prime} 5$ & 20 & 41 & 50 & 615 & 641 & 692 \\
\hline$C^{\prime} 1$ & 30 & 58 & 107 & 1090 & 1164 & 1173 \\
\hline$C^{\prime} 2$ & 30 & 130 & 157 & 1901 & 1909 & 1971 \\
\hline$C^{\prime} 3$ & 30 & 91 & 191 & 1745 & 1755 & 1832 \\
\hline$C^{\prime} 4$ & 30 & 89 & 172 & 1490 & $\frac{1554}{4}$ & 1573 \\
\hline$C^{\prime} 5$ & 30 & 79 & 95 & 1189 & 1229 & 1265 \\
\hline $\mathrm{D}^{\prime} 1$ & 35 & 167 & 172 & 2265 & $\overline{2265}$ & 2327 \\
\hline $\mathrm{D}^{\prime} 2$ & 35 & 130 & 274 & 2478 & 2502 & 2588 \\
\hline$D^{\prime} 3$ & 35 & 150 & 224 & 2287 & 2299 & 2333 \\
\hline$D^{\prime} 4$ & 35 & 186 & 129 & 2251 & $\overline{2251}$ & 2293 \\
\hline$D^{\prime} 5$ & 35 & 88 & 120 & 1405 & 1507 & 1616 \\
\hline$A^{\prime \prime} 1$ & 10 & 12 & 4 & 123 & 123 & 133 \\
\hline$A^{\prime \prime} 2$ & 10 & 18 & 5 & 161 & $\frac{161}{161}$ & 179 \\
\hline$A^{\prime \prime} 3$ & 10 & 15 & 3 & 142 & 142 & 160 \\
\hline$A^{\prime \prime} 4$ & 10 & 15 & 6 & 153 & $\overline{153}$ & 181 \\
\hline$A^{\prime \prime} 5$ & 10 & 10 & 3 & 101 & $\overline{115}$ & 103 \\
\hline $\mathrm{B}^{\prime \prime} 1$ & 20 & 77 & 46 & 897 & 897 & 924 \\
\hline $\mathrm{B}^{\prime \prime 2}$ & 20 & 80 & 34 & 883 & $\overline{883}$ & 904 \\
\hline $\mathrm{B}^{\prime \prime} 3$ & 20 & 54 & 25 & 590 & $\frac{200}{590}$ & 625 \\
\hline$B^{\prime \prime 4}$ & 20 & 101 & 22 & 969 & $\overline{969}$ & 969 \\
\hline$B^{\prime \prime} 5$ & 20 & 66 & 27 & 663 & 663 & 681 \\
\hline$C^{\prime \prime \prime} 1$ & 30 & 128 & 72 & 1473 & $\frac{1 \overline{473}}{4}$ & 1504 \\
\hline$C^{\prime \prime 2}$ & 30 & 203 & 53 & 1985 & 1985 & 2005 \\
\hline$C^{\prime \prime} 3$ & 30 & 204 & 87 & 2129 & 2129 & 2147 \\
\hline$C^{\prime \prime} 4$ & 30 & 134 & 74 & 1433 & 1433 & 1466 \\
\hline$C^{\prime \prime 5}$ & 30 & 115 & 54 & 1158 & 1158 & $\llbracket 192$ \\
\hline $\mathrm{D}^{\prime \prime} 1$ & 35 & 188 & 44 & 1857 & 1857 & 1871 \\
\hline $\mathrm{D}^{\prime \prime 2}$ & 35 & 266 & 114 & 2802 & 2802 & 2828 \\
\hline$D^{\prime \prime} 3$ & 35 & 182 & 32 & 1627 & 1627 & 1653 \\
\hline $\mathrm{D}^{\prime \prime 4}$ & 35 & 255 & 131 & 2618 & 2618 & 2645 \\
\hline $\mathrm{D}^{\prime \prime} 5$ & 35 & 189 & 120 & 2105 & 2105 & 2123 \\
\hline
\end{tabular}

Table 2. Performance comparisons between MIXED1 and MIXED2 (60 problems)

\begin{tabular}{lcccc}
\hline Problem sets & $\begin{array}{c}\text { Problem } \\
\text { characteristics }\end{array}$ & $\begin{array}{c}\text { Algorithm } \\
\text { MIXED1 }\end{array}$ & $\begin{array}{c}\text { Number } \\
\text { of ties }\end{array}$ & $\begin{array}{c}\text { Algorithm } \\
\text { MIXED2 }\end{array}$ \\
\hline A, B, C, D & $70 \%<P \leqslant 100 \%$ & 2 & 0 & 18 \\
$A^{\prime}, B^{\prime}, C^{\prime}, D^{\prime}$ & $40 \%<P \leqslant 70 \%$ & 17 & 0 & 3 \\
$A^{\prime \prime}, B^{\prime \prime}, C^{\prime \prime}, D^{\prime \prime}$ & $0 \%<P \leqslant 40 \%$ & 18 & 1 & 1 \\
\hline
\end{tabular}


and therefore form cycles. From previous experiments we found that this situation occurs, particularly, when $P=|A| /(|A|+|E|)$ is large. Obviously, such artificial cycles may be removed and solutions can be improved. This motivates the following modifications.

\subsection{Modified MIXED1 algorithm}

Phase I. Same as MIXED1.

Phase II. Same as MIXED1.

Phase III. Same as MIXED1.

Phase IV. Remove artificial cycles from $G_{4}$.

Let $A^{*}$ be the set of artificial arcs generated from Phases I, II, and III. Arbitrarily select an arc $(i, j)$ from $A^{*}$. Find a cycle (or a closed circuit) consisting of arcs from $A^{*}$ covering $(i, j)$. Update $A^{*}$ by removing the cycle (circuit) from $A^{*}$. Repeat this procedure until no more cycles (or closed circuits) can be found.

Finding artificial cycles (or closed circuits) covering a specified arc requires a shortest-path algorithm which is of $O\left(|V|^{2}\right)$ (see Christofides [15] and Pearn [16] for such procedures). Therefore, the complexity of Modified MIXEDI is the same as that of MIXED1. For the MCPP with six nodes, six edges, and two arcs described in Example 1, the Modified MIXED1 algorithm removes the following artificial cycle $\{(1,3),(3,2),(2,1)\}$ generating a solution of $6 M+7 \varepsilon$. Comparing this solution with that of MIXED1 which has a total length of $8 M+8 \varepsilon$, the improvement is significant (particularly, when $M \gg \varepsilon$ ).

\subsection{Modified MIXED2 algorithm}

Recalling that in Phase II of MIXED2 algorithm the minimal-cost matching algorithm was applied over the subnetwork containing edges only. If the lengths of the edges are relatively large, MIXED2 may perform poorly. In attempting to improve the solution, we took an alternative approach by first duplicating some arcs, and assigning directions to edges to convert the network into an Eulerian one.

Phase I. Same as MIXED2.

Phase II. Convert $G_{2}$ into an Eulerian network.

Step 1. Solve the CPP over the subnetwork consisting of $E_{2}$ and the associated nodes using the matching algorithm of Edmonds and Johnson [1]. The shortest distance between each pair of nodes, however, was calculated from the original (mixed) network with all arc directions ignored.

Step 2. For each pair of nodes $i$ and $j$ in the matching solution, the shortest path between $i$ and $j$ (may be directed in this case) is added to $G_{2}$. If the added path is directed, then clearly other edges in $E_{2}$ must be oriented to maintain the resulting network symmetric.

The complexity of the Modified MIXED2 algorithm is the same as that of MIXED2. Applying this modified algorithm over the MCPP described in Example 2, the optimal solution, with a total length of $6 M+12 \varepsilon$, can be obtained. In applying the matching algorithm, all arc directions have been ignored. Consequently, the following artificial edges $\{(1,2),(3,4),(5,6)\}$ rather than $\{(1,7),(7,2),(3,9),(9,4),(5,8),(8,6)\}$ were generated resulting in a cost reduction of $6 M-3 \varepsilon$. Following the same idea described in Frederickson [5], we can also consider a mixed-strategy approach. That is, we first call Modified MIXED1, and Modified MIXED2, then select the best of the two solutions. We refer to this approach as Modified MIXED1-2.

\section{COMPUTATIONAL RESULTS}

For the purpose of testing the proposed modifications and comparing them with the original solution procedures, we ran the 60 test problems which have been tested in Section 2. Table 3 represents the solutions generated by the four algorithms, MIXED1; MIXED2, Modified MIXED1, and Modified MIXED2 on the 60 test problems with emphasizes indicating the improved solutions. 
Table 3. Solution of the 60 test problems (underlines indicate the solutions improved)

\begin{tabular}{|c|c|c|c|c|c|c|}
\hline $\begin{array}{l}\text { Problem } \\
\text { number }\end{array}$ & $\begin{array}{l}\text { Number } \\
\text { of nodes }\end{array}$ & $\begin{array}{l}\text { Lower } \\
\text { bound }\end{array}$ & MIXEDI & $\begin{array}{l}\text { Modified } \\
\text { MIXEDI }\end{array}$ & MIXED2 & $\begin{array}{l}\text { Modified } \\
\text { MIXED2 }\end{array}$ \\
\hline Al & 10 & 120 & 192 & 192 & 177 & 168 \\
\hline A2 & 10 & 170 & 284 & 284 & 274 & $\overline{272}$ \\
\hline A3 & 10 & 100 & 186 & 165 & 154 & $\overline{154}$ \\
\hline A4 & 10 & 200 & 282 & 259 & 264 & 259 \\
\hline A5 & 10 & 110 & 162 & 139 & 137 & $\overline{135}$ \\
\hline B1 & 20 & 409 & 613 & 574 & 572 & 570 \\
\hline B2 & 20 & 426 & 658 & $\overline{642}$ & 619 & $\overline{610}$ \\
\hline B3 & 20 & 507 & 633 & 633 & 615 & $\frac{\overline{613}}{4}$ \\
\hline B4 & 20 & 636 & 824 & 811 & 814 & 810 \\
\hline B5 & 20 & 604 & 806 & 802 & 787 & $\overline{787}$ \\
\hline $\mathrm{C} 1$ & 30 & 1186 & 1354 & $1 \overline{354}$ & 1349 & 1339 \\
\hline $\mathrm{C} 2$ & 30 & 1153 & 1337 & 1323 & 1324 & $\overline{1323}$ \\
\hline C3 & 30 & 1587 & 1787 & 1787 & 1769 & $\frac{25}{1763}$ \\
\hline C4 & 30 & 1182 & 1230 & 1230 & 1261 & 1225 \\
\hline C5 & 30 & 801 & 1075 & 1040 & 1028 & $\frac{1025}{1025}$ \\
\hline DI & 35 & 2133 & 2195 & 2189 & 2225 & $\frac{1020}{2200}$ \\
\hline D2 & 35 & 1837 & 2045 & $\overline{2045}$ & 2030 & 2023 \\
\hline D3 & 35 & 1505 & 1861 & 1826 & 1798 & 1798 \\
\hline D4 & 35 & 994 & 1436 & 1362 & 1331 & 1330 \\
\hline D5 & 35 & 1537 & 1727 & 1694 & 1686 & 1684 \\
\hline$A^{\prime} 1$ & 10 & 185 & 185 & 185 & 207 & 200 \\
\hline$A^{\prime} 2$ & 10 & 110 & 180 & 157 & 157 & 157 \\
\hline$A^{\prime} 3$ & 10 & 167 & 213 & 200 & 205 & 192 \\
\hline$A^{\prime} 4$ & 10 & 209 & 229 & $\overline{229}$ & 254 & $\overline{222}$ \\
\hline$A^{\prime} 5$ & 10 & 181 & 217 & 217 & 220 & 211 \\
\hline $\mathbf{B}^{\prime} \mathbf{1}$ & 20 & 485 & 545 & 545 & 558 & 548 \\
\hline $\mathbf{B}^{\prime} 2$ & 20 & 757 & 763 & 763 & 782 & $\overline{769}$ \\
\hline $\mathbf{B}^{\prime} 3$ & 20 & 631 & 685 & 685 & 671 & $\frac{766}{666}$ \\
\hline$B^{\prime} 4$ & 20 & 547 & 585 & 585 & 579 & 566 \\
\hline $\mathbf{B}^{\prime} 5$ & 20 & 615 & 641 & 641 & 692 & $\overline{649}$ \\
\hline$C^{\prime} 1$ & 30 & 1090 & 1164 & 1164 & 1173 & 1160 \\
\hline$C^{\prime} 2$ & 30 & 1901 & 1909 & 1909 & 1971 & 1922 \\
\hline$C^{\prime} 3$ & 30 & 1745 & 1755 & 1755 & 1832 & $\overline{1780}$ \\
\hline$C^{\prime} 4$ & 30 & 1490 & 1554 & 1554 & 1573 & $\frac{1540}{150}$ \\
\hline$C^{\prime} 5$ & 30 & 1189 & 1229 & 1215 & 1265 & $\overline{1241}$ \\
\hline$D^{\prime} 1$ & 35 & 2265 & 2265 & $\overline{2265}$ & 2327 & $\overline{2296}$ \\
\hline $\mathrm{D}^{\prime} 2$ & 35 & 2478 & 2502 & 2502 & 2588 & $\overline{2522}$ \\
\hline$D^{\prime} 3$ & 35 & 2287 & 2299 & 2299 & 2333 & 2312 \\
\hline$D^{\prime} 4$ & 35 & 2251 & 2251 & 2251 & 2293 & 2290 \\
\hline$D^{\prime} 5$ & 35 & 1405 & 1507 & 1507 & 1616 & 1532 \\
\hline $\mathbf{A}^{n} \mathbf{1}$ & 10 & 123 & 123 & 123 & 133 & 123 \\
\hline$A^{\prime \prime} 2$ & 10 & 161 & 161 & 161 & 179 & $\overline{161}$ \\
\hline$A^{\prime \prime} 3$ & 10 & 142 & 142 & 142 & 160 & 147 \\
\hline$A^{\prime \prime} 4$ & 10 & 153 & 153 & 153 & 181 & 158 \\
\hline$A^{\prime \prime} 5$ & 10 & 101 & 115 & 115 & 103 & $\overline{103}$ \\
\hline$B^{\prime \prime} 1$ & 20 & 897 & 897 & 897 & 924 & 915 \\
\hline$B^{\prime \prime 2} 2$ & 20 & 883 & 883 & 883 & 904 & $\overline{893}$ \\
\hline$B^{\prime \prime} 3$ & 20 & 590 & 590 & 590 & 625 & 611 \\
\hline$B^{\prime \prime} 4$ & 20 & 969 & 969 & 969 & 969 & 969 \\
\hline$B^{\prime \prime} 5$ & 20 & 663 & 663 & 663 & 681 & 679 \\
\hline$C^{\prime \prime} 1$ & 30 & 1473 & 1473 & 1473 & 1504 & $1 \overline{491}$ \\
\hline $\mathrm{C}^{\prime \prime} 2$ & 30 & 1985 & 1985 & 1985 & 2005 & 1999 \\
\hline$C^{\prime \prime} 3$ & 30 & 2129 & 2129 & 2129 & 2147 & 2143 \\
\hline $\mathrm{C}^{\prime \prime} 4$ & 30 & 1433 & 1433 & 1433 & 1466 & 1450 \\
\hline$C^{\prime \prime} 5$ & 30 & 1158 & 1158 & 1158 & 1192 & 1186 \\
\hline $\mathrm{D}^{\prime \prime} 1$ & 35 & 1857 & 1857 & 1857 & 1871 & $\frac{1871}{187}$ \\
\hline $\mathrm{D}^{\prime \prime 2}$ & 35 & 2802 & 2802 & 2802 & 2828 & 2814 \\
\hline$D^{\prime \prime 3}$ & 35 & 1627 & 1627 & 1627 & 1653 & $\overline{1650}$ \\
\hline$D^{\prime \prime 4}$ & 35 & 2618 & 2618 & 2618 & 2645 & $\overline{2642}$ \\
\hline$D^{\prime \prime 5}$ & 35 & 2105 & 2105 & 2105 & 2123 & $\overline{2118}$ \\
\hline
\end{tabular}

\section{Comparisons between modified MIXEDI and modified MIXED2}

In Table 4, the performance of Modified MIXED1 and Modified MIXED2 are compared. The results obtained were quite similar to that for comparing MIXED1 and MIXED2:

(1) Modified MIXED1 significantly outperformed Modified MIXED2 for problems with $0 \%<P \leqslant 70 \%$, as Modified MIXED1 received 28 best solutions out of 40 test problems (which is $70 \%$ );

(2) Modified MIXED2 significantly outperformed Modified MIXED1 for problems with $70 \%<P \leqslant 100 \%$, as Modified MIXED received 17 best solutions out of 20 test problems (which is $85 \%$ ). 
Table 4. Performance comparisons between Modified MIXED1 and Modified MIXED2 (70 problems)

\begin{tabular}{lcccc}
\hline Problem sets & $\begin{array}{c}\text { Problem } \\
\text { characteristics }\end{array}$ & $\begin{array}{c}\text { Modified } \\
\text { MIXED1 }\end{array}$ & $\begin{array}{c}\text { Number } \\
\text { of ties }\end{array}$ & $\begin{array}{r}\text { Modified } \\
\text { MIXED2 }\end{array}$ \\
\hline A, B, C, D & $70 \%<P \leqslant 100 \%$ & 1 & 2 & 17 \\
A $^{\prime}, B^{\prime}, C^{\prime}, D^{\prime}$ & $40 \%<P \leqslant 70 \%$ & 12 & 1 & 7 \\
A $^{\prime \prime}, B^{\prime \prime}, C^{\prime \prime}, D^{\prime \prime}$ & $0 \%<P \leqslant 40 \%$ & 16 & 3 & 1 \\
\hline
\end{tabular}

Table 5. Percentage of problem solutions improved by the modifications ( 60 problems)

\begin{tabular}{lcccc}
\hline Problem sets & $\begin{array}{c}\text { Problem } \\
\text { characteristics }\end{array}$ & $\begin{array}{c}\text { Modified } \\
\text { MIXED1 }\end{array}$ & $\begin{array}{c}\text { Modified } \\
\text { MIXED2 }\end{array}$ & $\begin{array}{c}\text { Modified } \\
\text { MIXED1-2 }\end{array}$ \\
\hline A, B, C, D & $70 \%<P \leqslant 100 \%$ & $65 \%$ & $85 \%$ & $85 \%$ \\
$A^{\prime}, B^{\prime}, C^{\prime}, D^{\prime}$ & $40 \%<P \leqslant 70 \%$ & $15 \%$ & $95 \%$ & $40 \%$ \\
$A^{\prime \prime}, B^{\prime \prime}, C^{\prime \prime}, D^{\prime \prime}$ & $0 \%<P \leqslant 40 \%$ & $0 \%$ & $85 \%$ & $0 \%$ \\
\hline
\end{tabular}

\section{Comparisons between modified approaches and the original algorithms}

In Table 5, the performance between the proposed modifications and the original algorithms are compared in terms of the percentage of problem solutions improved. We note that:

(1) modified MIXED1 improved MIXED1 for $65 \%$ of problems with $70 \%<P \leqslant 100 \%$, $15 \%$ of problems with $40 \%<P \leqslant 70 \%$, and no improvement for problems with $0 \%<P \leqslant 40 \%$ (we note that for 19 out of 20 problems in this category, MIXED1 obtained problem optimal solutions; therefore, very little improvement can be made);

(2) modified MIXED2 improved MIXED2 for $85 \%$ of problems with $70 \%<P \leqslant 100 \%$, $95 \%$ of problems with $40 \%<P \leqslant 70 \%$, and $85 \%$ for problems with $0 \%<P \leqslant 40 \%$. The improvement, clearly, is very significant.

For mixed strategies, Modified MIXED1-2 improved MIXED1-2 for $85 \%$ of the problems with $70 \%<P \leqslant 100 \%, 40 \%$ for problems with $40 \%<P \leqslant 70 \%$, and no improvement for problems with $0 \%<P \leqslant 40 \%$. We point out that this comparison is important since the two mixed strategies outperformed their corresponding MIXED1 and MIXED2 procedures. In Table 6, the performance of the four algorithms are compared in terms of average deviation from the lower bound, number of problems receiving the best solutions among all algorithms, number of problems achieving the problem lower bounds (in this case, the solution obtained is optimal), and the worst solution in terms of percentage above the lower bound. The results indicated that:

(1) for problems with $40 \%<P \leqslant 70 \%$ and $70 \%<P \leqslant 100 \%$, Modified MIXED1 improved MIXED1 for about 1.5 and $4.4 \%$, respectively;

(2) for problems with $0 \%<P \leqslant 40 \%, 40 \%<P \leqslant 70 \%$, and $70 \%<P \leqslant 100 \%$, Modified MIXED2 improved MIXED2 for about $2.6 \%$, 3.4\%, and $1.1 \%$, respectively;

(3) for problems with $0 \%<P \leqslant 40 \%$, MIXED1 performed very well as the algorithm received 19 problem optimal solutions out of 20 test problems (which is $95 \%$ ). Consequently, no further improvements can be made by modified MIXED1;

(4) for problems with large $P(70 \%<P \leqslant 100 \%)$, none of the four algorithms seem to work well (the average percentages above the lower bound for the four algorithms all exceed $25 \%$ ). This is owing to the fact that our lower bounds were obtained from solving the CPP over the (undirected) network derived from the original one by ignoring all the arc directions.

In our testing, the run times for problems of the same size are very much the same. Therefore, instead of showing the average run time, we only display (Table 7) six test problems in CPU seconds on the IBM 486 as an indication of the relative efficiency of the four algorithms. We note that all the four algorithms are very efficient. A problem with 35 nodes, and 350 edges and arcs only takes less than $2 \mathrm{CPU}$ seconds. It should be pointed out that the run times difference between the modified 
Table 6. Performance comparisons of the four algorithms (60 problems)

\begin{tabular}{|c|c|c|c|c|c|}
\hline & $\begin{array}{c}\text { Problem } \\
\text { characteristics }\end{array}$ & MIXED1 & $\begin{array}{l}\text { Modified } \\
\text { MIXED1 }\end{array}$ & MIXED2 & $\begin{array}{l}\text { Modified } \\
\text { MIXED2 }\end{array}$ \\
\hline $\begin{array}{l}\text { Average percentage above } \\
\text { the lower bound }\end{array}$ & $\begin{aligned} 70 \% & <P \leqslant 100 \% \\
40 \% & <P \leqslant 70 \% \\
0 \% & <P \leqslant 40 \%\end{aligned}$ & $\begin{array}{r}33.46 \\
8.89 \\
0.69\end{array}$ & $\begin{array}{r}29.10 \\
7.39 \\
0.69\end{array}$ & $\begin{array}{r}26.80 \\
10.89 \\
4.03\end{array}$ & $\begin{array}{l}25.69 \\
7.5 \\
1.41\end{array}$ \\
\hline $\begin{array}{l}\text { Number of problems } \\
\text { receiving the best } \\
\text { solution }\end{array}$ & $\begin{aligned} 70 \% & <P \leqslant 100 \% \\
40 \% & <P \leqslant 70 \% \\
0 \% & <P \leqslant 40 \%\end{aligned}$ & $\begin{array}{r}0 \\
11 \\
19\end{array}$ & $\begin{array}{r}3 \\
13 \\
19\end{array}$ & $\begin{array}{l}4 \\
1 \\
2\end{array}$ & $\begin{array}{r}19 \\
8 \\
4\end{array}$ \\
\hline $\begin{array}{l}\text { Number of problems } \\
\text { achieving the lower } \\
\text { bound }\end{array}$ & $\begin{aligned} 70 \% & <P \leqslant 100 \% \\
40 \% & <P \leqslant 70 \% \\
0 \% & <P \leqslant 40 \%\end{aligned}$ & $\begin{array}{r}0 \\
3 \\
19\end{array}$ & $\begin{array}{r}0 \\
3 \\
19\end{array}$ & $\begin{array}{l}0 \\
0 \\
1\end{array}$ & $\begin{array}{l}0 \\
0 \\
3\end{array}$ \\
\hline $\begin{array}{l}\text { Worst solution among } \\
60 \text { problems in term of } \\
\text { percentage above the } \\
\text { lower bound }\end{array}$ & $\begin{aligned} 70 \% & <P \leqslant 100 \% \\
40 \% & <P \leqslant 70 \% \\
0 \% & <P \leqslant 40 \%\end{aligned}$ & $\begin{array}{l}86.00 \\
63.64 \\
13.86\end{array}$ & $\begin{array}{l}67.06 \\
42.73 \\
13.86\end{array}$ & $\begin{array}{l}61.18 \\
42.73 \\
18.30\end{array}$ & $\begin{array}{r}60.00 \\
42.73 \\
3.56\end{array}$ \\
\hline
\end{tabular}

Table 7. Run time comparisons (in CPU s) of the four algorithms

\begin{tabular}{|c|c|c|c|c|c|c|c|}
\hline $\begin{array}{l}\text { Problem } \\
\text { number }\end{array}$ & $\begin{array}{l}\text { Number } \\
\text { of nodes }\end{array}$ & $\begin{array}{l}\text { Number } \\
\text { of edges }\end{array}$ & $\begin{array}{l}\text { Number } \\
\text { of arcs }\end{array}$ & MIXED1 & MIXED2 & $\begin{array}{l}\text { Modified } \\
\text { MIXED1 }\end{array}$ & $\begin{array}{l}\text { Modified } \\
\text { MIXED2 }\end{array}$ \\
\hline B1 & 20 & 7 & 59 & 0.110 & 0.055 & 0.110 & 0.055 \\
\hline $\mathbf{B}^{\prime} 1$ & 20 & 28 & 40 & 0.110 & 0.165 & 0.110 & 0.165 \\
\hline$B^{\prime \prime} 1$ & 20 & 77 & 46 & 0.165 & 0.165 & 0.165 & 0.165 \\
\hline $\mathrm{C} 1$ & 35 & 57 & 323 & 1.879 & 0.824 & 0.879 & 0.824 \\
\hline$C^{\prime} 1$ & 35 & 167 & 172 & 1.154 & 1.099 & 1.154 & 1.099 \\
\hline$C^{\prime \prime} 1$ & 35 & 188 & 44 & 0.604 & 0.549 & 0.604 & 0.549 \\
\hline
\end{tabular}

approaches and the original solution procedures for the same test problem, is negligible (less than 0.055 CPU seconds). Therefore, the difference does not show in Table 7.

\section{CONCLUSIONS}

In this paper, we presented two efficient modifications of the existing algorithms (MIXED1, MIXED2), which we referred to as Modified MIXED1 and Modified MIXED2. The two modified procedures run very fast, and work well in general. We have tested them on many problems which were arbitrarily generated. The computational results indicated that: (1) modified MIXED1 improved MIXED1 for $65 \%$ of problems with $70 \%<P \leqslant 100 \%$, and $15 \%$ of problems with $40 \%<P \leqslant 70 \%$, (2) Modified MIXED2 improved MIXED2 for $85 \%$ of problems with $70 \%<P \leqslant 100 \%, 95 \%$ of problems with $40 \%<P \leqslant 70 \%$, and $85 \%$ of problems with $0 \%<P \leqslant 40 \%$. For mixed strategies which are the procedures of choice, Modified MIXED1-2 improved MIXED1-2 for $85 \%$ of the problems with $70 \%<P \leqslant 100 \%$, and $40 \%$ of problems with $40 \%<P \leqslant 70 \%$.

\section{REFERENCES}

1. J. Edmonds and E. Johnson, Matching, Euler tours and the Chinese postman. Math. Progr. 5, 88-124 (1973).

2. C. H. Papadimitriou, On the complexity of edge traversing. J. Assoc. Comput. Mach. 23, 544-554 (1976).

3. N. Christofides, E. Benavent, V. Campos, A. Corbran and E. Mota, An optimal method for the mixed postman problem. Proceedings of the 11th IFIP Conference, Copenhagen, Denmark, pp. $641-649$ (1983).

4. E. Minieka, The Chinese postman problem for mixed networks. Mgmnt Sci. 25, 643-648 (1979).

5. G. N. Frederickson, Approximation algorithms for some postman problems. J. Assoc. Comput. Mach. 26, 538-554 (1979).

6. P. Brucker, The Chinese postman problem for mixed graphs. Lecture Notes Comput. Sci. 100, 354-366 (1981).

7. L. Levy and L. Bodin, Scheduling in the postal carriers for the United States postal service: an application of arc partitioning and routing. In Vehicle Routing: Methods and Studies, pp. 359-394. North-Holland, Amsterdam (1988).

8. J. N. Holt and A. M. Watts, Vehicle routing and scheduling in the newspaper industry. In Vehicle Routing: Methods and Studies, pp. 347-358. North-Holland, Amsterdam (1988).

9. E. Beltrami and L. Bodin, Networks and vehicle routing for municipal waste collection. Networks 4, 65-94 (1974). 
10. J. Desrosiers, J. A. Ferland, J. M. Rousseau, G. Lapalme and L. Chapleau, An overview of a school busing system. In Scientific Management of Transport Systems, pp. 235-243. North-Holland, Amsterdam (1988).

11. R. W. Eglese and L. Y. O. Li, Efficient routeing for winter gritting. J. Opl Res. Soc. 43, 1031-1034 (1992).

12. R. W. Eglese, Routeing winter gritting vehicles, to appear in Discrete Appl. Math. (1992).

13. H. I. Stern and M. Dror, Routing electric meter readers. Comput. Ops Res. 6, 209-223 (1979).

14. A. Nijenhuis and H. S. Wilf, Combinatorial Algorithms. Academic Press, New York (1978).

15. N. Christofides, The optimal traversal of a graph. Omega 1, 719-732 (1973).

16. W. L. Pearn, Approximate solutions for the capacitated arc routing problem. Comput. Ops Res. 16, 589-600 (1989). 